\title{
Radioembolization for Metastatic Colorectal Cancer
}

\author{
David Guez, MD ${ }^{1}$ Patrick D. Sutphin, MD, $\mathrm{PhD}^{20}$ \\ ${ }^{1}$ Division of Interventional Radiology, Department of Radiology, \\ Boston Medical Center, Boston University School of Medicine, \\ Boston, Massachusetts \\ 2 Division of Interventional Radiology, Department of Radiology, \\ Massachusetts General Hospital, Harvard Medical School, Boston, \\ Massachusetts
}

Dig Dis Interv 2021;5:90-92.

\author{
Suvranu Ganguli, MD ${ }^{1 \odot}$
}

\begin{abstract}
Keywords

- radioembolization

- colorectal cancer

- transarterial therapy
\end{abstract}

\begin{abstract}
Address for correspondence David Guez, MD, Division of Interventional Radiology, Department of Radiology, Boston Medical Center, Boston University School of Medicine, 840 Harrison Avenue, 1st floor, Boston, MA 02118 (e-mail: David.guez@bmc.org).
\end{abstract}

The liver is the most common site of metastatic disease in colorectal cancer, and, in the setting of liver-dominant disease, a chief contributor to mortality. ${ }^{1,2}$ The natural history of metastatic colorectal cancer (mCRC) without treatment is progression of disease with a median survival of approximately 4.5 months in patients with synchronous liver metastases. ${ }^{3}$

Chemotherapy is the backbone of treatment for mCRC, with modern chemotherapy regimens achieving a median survival of 30 months in some clinical trials. This represents a substantial increase in survival over the last two decades, ${ }^{4}$ with the 2-year survival rate in patients with mCRC of $21 \%$ in the mid-1990s to $37 \%$ from 2009 to $2015 .{ }^{5}$ However, except for a small fraction of patients on immunotherapy, the duration of response is limited and resistance to therapy inevitably develops. As hepatic metastases contribute significantly to patient mortality, nonsystemic treatment options that directly address liver metastases offer the possibility of extending life. Proof of concept of this benefit is evident in the small fraction of patients, estimated at 10 to $20 \%$, eligible for surgical resection of hepatic metastases with 10-year survival rates up to $20 \%$ after resection. ${ }^{6}$

Only a minority of patients with colorectal liver metastases are candidates for surgical resection; thus, alternative means

received

January 7, 2021 accepted after revision April 1, 2021
T. Gandhi, MD, FSIR, FSVM and Suvranu Ganguli, MD, FSIR must be considered for the treatment of liver metastases. It has been demonstrated in necropsy specimens, and then corroborated with in vivo studies, that the dominant blood supply to liver metastases is via the hepatic artery. ${ }^{7,8}$ This predominant hepatic arterial supply of metastatic hepatic lesions may be used for both diagnostic and therapeutic purposes. Radioembolization can be used to exploit this, via catheter-directed intra-arterial administration of a radioactive isotope, delivered attached to a microsphere, with intent for therapeutic effect on hepatic tumors. In the setting of $\mathrm{mCRC}$, radioembolization with yttrium-90 $\left({ }^{90} \mathrm{Y}\right)$ is the most commonly used isotope for liver-directed therapy, with the most available clinical data.

\section{Patient Selection}

According to the National Comprehensive Cancer Network (NCCN), radioembolization with ${ }^{90} \mathrm{Y}$ is an appropriate option in patients with liver-dominant, chemotherapy-resistant mCRC. Absolute contraindications include excessive hepatopulmonary shunting which may lead to radiation-induced pneumonitis or pulmonary fibrosis, and gastrointestinal deposition of microspheres which can lead to ulceration. Relative contraindications include uncorrectable coagulopathy, iodinated (c) 2021. Thieme. All rights reserved. Thieme Medical Publishers, Inc., 333 Seventh Avenue, 18th Floor, New York, NY 10001, USA
DOI https://doi.org/ 10.1055/s-0041-1729944. ISSN 2472-8721. 
contrast allergy, poor kidney/liver function, and Eastern Cooperative Oncology Group (ECOG) performance status greater than 2 .

Studies have demonstrated various predictors of increased survival following radioembolization for MCRC, including ECOG $0,{ }^{9}$ albumin greater than $3 \mathrm{~g} / \mathrm{dL},{ }^{9}$ hepatic tumor burden $\leq 25 \%$ and no extrahepatic disease, ${ }^{9-12} \geq 30 \%$ response rate of carcinoembryonic antigen (CEA) at 3 months posttreatment, ${ }^{11}$ response on positron emission tomography (PET) scan, ${ }^{13}$ and $\leq 2$ prior chemotherapy agents. ${ }^{9-12}$ Predictors of decreased survival following radioembolization include uncontrolled ascites, KRAS mutation, CEA greater than $62 \mathrm{ng} / \mathrm{mL}$, low ADC on magnetic resonance imaging, and lymphovascular invasion of primary tumor. ${ }^{14}$ In one study, a lung shunt fraction of greater than $10 \%$ was associated with decreased median overall survival (OS) of 6.9 versus 10.0 months. $^{15}$

\section{Clinical Data}

\section{Radioembolization as First-Line Therapy}

The SIRFLOX trial was the first Phase III prospective, open-label, multinational, randomized controlled trial to include radioembolization in first-line treatment of mCRC. A total of 530 patients were randomized, with 263 receiving FOLFOX with or without bevacizumab, and 267 receiving the same regimen in addition to radioembolization with resin microsphere. Trial participants needed to have liver only, or liver-dominant, disease, and were fit for combo therapy with radioembolization. The primary endpoint was progression-free survival(PFS). Secondary endpoints were PFS-L(PFS in the liver), OS, response rate, quality of life, recurrence rate, toxicity and safety, and resection rate. Of the 267 patients who received radioembolization, 145 received bevacizumab. The study did not meet the primary endpoint, as PFS was 10.7 months with radioembolization and 10.2 months for the control arm $(p=0.43)$. It is worth consideration that $40 \%$ of patients in the radioembolization arm had extrahepatic disease and $45 \%$ did not have the primary tumor removed. Additionally, objective response rate in the liver was $79 \%$ in the radioembolization arm versus $69 \%$ in the control arm $(p=0.042)$, and PFS-L demonstrated a 7.9-month improvement in the radioembolization arm over the control arm (20.5 vs. 12.6 months, $p=0.002)^{16}$

The combined analysis of the three randomized, multicenter, Phase III trials of SIRFLOX, FOXFIRE, and FOXFIRE Global looked at 1,103 patients separated into chemotherapy versus chemotherapy plus radioembolization with resin. Response and hepatic free progression favored ${ }^{90} \mathrm{Y}$; however, there was no difference in OS and PFS. Subset analysis did demonstrate a 4.9-month survival benefit for patients with right-sided primary tumor; however, based on the findings, they could not recommend first-line use of ${ }^{90} \mathrm{Y}$ in combination with chemotherapy in unselected patients with mCRC. ${ }^{17}$ There were some criticisms associated with these studies. Only 84\% of the patients who were allocated to receive ${ }^{90} \mathrm{Y}$ actually received ${ }^{90} \mathrm{Y}$ (7.9\% did not receive ${ }^{90} \mathrm{Y} ; 7.7 \%$ with bilobar disease received unilobar therapy only), and increased liver response did not directly lead to increased resection. It is unclear if there was extrahepatic progression in these patients or could there have been a reluctance to operate post- ${ }^{90} \mathrm{Y}$ ? Additional criticism included that some of the participating centers involved in the SIRFLOX study had very limited experience with radioembolization. ${ }^{18}$

\section{Radioembolization as Second-Line Therapy}

The EPOCH trial is intended to evaluate the efficacy of glass microspheres in patients with $\mathrm{mCRC}$ who are scheduled to receive second-line chemotherapy. This study is a randomized, open-label trial that has enrolled 428 patients set up to receive standard-of-care chemotherapy with or without radioembolization, with enrollment recently completed. Primary endpoints will include PFS and PFS-L. ${ }^{19}$ The results from this trial will be highly anticipated, as most patients who receive first-line chemotherapy will require further treatment.

\section{Radioembolization as Salvage Therapy}

Between 2005 and 2010, six studies evaluated radioembolization of colorectal liver metastases as third-line treatment after FOLFOX and FOLFIRI, demonstrating similar results in the salvage setting (meaning third line and beyond). In each retrospective study, between 27 and 208 patients were treated with radioembolization in the salvage setting; response rate via RECIST was 35 to $40 \%$, metabolic response via PET was 80 to $90 \%$, and median OS was between 7.9 and 14.5 months. $^{20-25}$

In 2017, the MORE (Metastatic colorectal cancer liver metastases Outcomes after RadioEmbolization) study published the updated survival analysis of its retrospective review of 606 patients with liver-only or liver-dominant mCRC, not deemed suitable for surgery, ablation, or systemic therapy, and which had progressed or become refractory to at least one line of systemic therapy. These patients received radioembolization with resin microspheres between 2002 and 2011 at one of 11 U.S. tertiary care centers. Updated results demonstrated a median OS of 10.0 months, ${ }^{12}$ echoing similar results demonstrated in the studies mentioned previously. Key findings from subgroup analysis demonstrated no difference in OS or toxicity in patients older than 70 years versus patients younger than 70 years, indicating radioembolization is well tolerated and effective, even in the elderly. Additional factors found to be significantly associated with poor survival included anemia (hemoglobin $<10.0 \mathrm{~g} / \mathrm{dL}$ ), baseline liver dysfunction (presence of ascites, abnormal levels of albumin, alkaline phosphatase, aspartate aminotransferase, and bilirubin), and lung shunt fraction greater than $10 \%$. Baseline characteristics and treatment-related factors significantly associated with survival of more than 1 year included hemoglobin levels greater than $10 \mathrm{~g} / \mathrm{dL}$, adequate liver function, and less than two prior lines of chemotherapy. Hickey et al published similar median OS of 10.6 months in a retrospective study examining 531 patients across eight institutions who exclusively received radioembolization with glass microspheres in the setting of $\mathrm{mCRC}{ }^{9}$ They found performance status, less than $25 \%$ hepatic tumor burden, no extrahepatic metastases (median OS in the setting of no extrahepatic mets 
was 14.4 months [ $n=329$ patients], $p<0.001$ ), serum albumin greater than $3 \mathrm{~g} / \mathrm{dL}$, and two or less prior lines of chemotherapy to be independent predictors of better survival outcomes. Additionally, they found the procedure to be well tolerated, with fatigue (55\%), abdominal pain (34\%), and nausea (19\%) to be the most common adverse events. Per the authors, "Grade 3 or 4 biochemical toxicity, recorded at any time after treatment, included effects on the level of bilirubin (13\%), alkaline phosphatase (9\%), albumin ( $8 \%)$, AST (3\%), ALT $(<1 \%)$." These studies demonstrate a consistent role for radioembolization as liver-directed salvage therapy in $\mathrm{mCRC}$, regardless of whether glass or resin microspheres are used.

\section{Conclusion}

Retrospective studies of liver-directed radioembolization have demonstrated consistent outcomes in the salvage setting for liver-dominant mCRC patients, regardless of whether glass or resin particles are used. Radioembolization carries a low adverse event profile, allowing patients of advanced age to tolerate the procedure and benefit from similar survival and toxicity endured by younger patients with similar disease. While level I evidence does not support first-line use of ${ }^{90} \mathrm{Y}$ in $\mathrm{mCRC}$, we await to see the results of the Phase III EPOCH trial that is currently underway in evaluating ${ }^{90} \mathrm{Y}$ as the second-line therapy.

\section{Conflict of Interest}

None declared.

\section{References}

1 Riihimäki M, Hemminki A, Sundquist J, Hemminki K. Patterns of metastasis in colon and rectal cancer. Sci Rep 2016;6:29765

2 Helling TS, Martin M. Cause of death from liver metastases in colorectal cancer. Ann Surg Oncol 2014;21(02):501-506

3 Bengtsson G, Carlsson G, Hafström L, Jönsson PE. Natural history of patients with untreated liver metastases from colorectal cancer. Am J Surg 1981;141(05):586-589

4 Kuipers EJ, Grady WM, Lieberman D, et al. Colorectal cancer. Nat Rev Dis Primers 2015;1:15065

5 Siegel RL, Miller KD, Goding Sauer A, et al. Colorectal cancer statistics, 2020. CA Cancer J Clin 2020;70(03):145-164

6 Wong SL, Mangu PB, Choti MA, et al. American Society of Clinical Oncology 2009 clinical evidence review on radiofrequency ablation of hepatic metastases from colorectal cancer. J Clin Oncol 2010;28(03):493-508

7 Wright RD. The blood supply of newly developed epithelial tissue in the liver. J Pathol Bacteriol 1937;45(02):405-414

8 Bierman HR, Byron RLJr, Kelley KH, Grady A. Studies on the blood supply of tumors in man. III. Vascular patterns of the liver by hepatic arteriography in vivo. J Natl Cancer Inst 1951;12(01): 107-131

9 Hickey R, Lewandowski RJ, Prudhomme T, et al. ${ }^{90} \mathrm{Y}$ radioembolization of colorectal hepatic metastases using glass microspheres: safety and survival outcomes from a 531-patient multicenter study. J Nucl Med 2016;57(05):665-671

10 Abbott AM, Kim R, Hoffe SE, et al. Outcomes of TheraSphere radioembolization for colorectal metastases. Clin Colorectal Cancer 2015;14(03):146-153
11 Jakobs TF, Paprottka KJ, Raeßler F, et al. Robust evidence for longterm survival with ${ }^{90} \mathrm{Y}$ radioembolization in chemorefractory liver-predominant metastatic colorectal cancer. Eur Radiol 2017;27(01):113-119

12 Kennedy A, Cohn M, Coldwell DM, et al. Updated survival outcomes and analysis of long-term survivors from the MORE study on safety and efficacy of radioembolization in patients with unresectable colorectal cancer liver metastases. J Gastrointest Oncol 2017;8(04):614-624

13 Kalva SP, Rana RS, Liu R, et al. Yttrium-90 radioembolization as salvage therapy for liver metastases from colorectal cancer. Am J Clin Oncol 2017;40(03):288-293

14 Boas FE, Bodei L, Sofocleous CT. Radioembolization of colorectal liver metastases: indications, technique, and outcomes. J Nucl Med 2017;58(Suppl 2):104S-111S

15 Narsinh KH, Van Buskirk M, Kennedy AS, et al. Hepatopulmonary shunting: a prognostic indicator of survival in patients with metastatic colorectal adenocarcinoma treated with ${ }^{90} \mathrm{Y}$ radioembolization. Radiology 2017;282(01):281-288

16 van Hazel GA, Heinemann V, Sharma NK, et al. SIRFLOX: randomized phase III trial comparing first-line mFOLFOX6 (plus or minus bevacizumab) versus mFOLFOX6 (plus or minus bevacizumab) plus selective internal radiation therapy in patients with metastatic colorectal cancer. J Clin Oncol 2016;34(15):1723-1731

17 Wasan HS, Gibbs P, Sharma NK, et al; FOXFIRE Trial Investigators SIRFLOX Trial Investigators FOXFIRE-Global Trial Investigators. First-line selective internal radiotherapy plus chemotherapy versus chemotherapy alone in patients with liver metastases from colorectal cancer (FOXFIRE, SIRFLOX, and FOXFIRE-Global): a combined analysis of three multicentre, randomised, phase 3 trials. Lancet Oncol 2017;18(09):1159-1171

18 Ali R, Gabr A, Mora R, Riaz A, Lewandowski R. Role of Y-90 radioembolization in hepatic metastatic colorectal carcinoma. In: Correia M, Choti M, Rocha F, Wakabayashi G, eds. Colorectal Cancer Liver Metastases. Cham: Springer; 2020

19 Chauhan N, Mulcahy MF, Salem R, et al. TheraSphere yttrium-90 glass microspheres combined with chemotherapy versus chemotherapy alone in second-line treatment of patients with metastatic colorectal carcinoma of the liver: protocol for the EPOCH Phase 3 randomized clinical trial. JMIR Res Protoc 2019;8(01): e11545

20 Lewandowski RJ, Thurston KG, Goin JE, et al. ${ }^{90} \mathrm{Y}$ microsphere (TheraSphere) treatment for unresectable colorectal cancer metastases of the liver: response to treatment at targeted doses of 135-150 Gy as measured by [18F]fluorodeoxyglucose positron emission tomography and computed tomographic imaging. J Vasc Interv Radiol 2005;16(12):1641-1651

21 Kennedy AS, Coldwell D, Nutting C, et al. Resin 90Y-microsphere brachytherapy for unresectable colorectal liver metastases: modern USA experience. Int J Radiat Oncol Biol Phys 2006;65(02): $412-425$

22 Jakobs TF, Hoffmann RT, Dehm K, et al. Hepatic yttrium-90 radioembolization of chemotherapy-refractory colorectal cancer liver metastases. J Vasc Interv Radiol 2008;19(08):1187-1195

23 Mulcahy MF, Lewandowski RJ, Ibrahim SM, et al. Radioembolization of colorectal hepatic metastases using yttrium-90 microspheres. Cancer 2009;115(09):1849-1858

24 Cianni R, Urigo C, Notarianni E, et al. Selective internal radiation therapy with SIR-spheres for the treatment of unresectable colorectal hepatic metastases. Cardiovasc Intervent Radiol 2009;32(06):1179-1186

25 Evans KA, Richardson MG, Pavlakis N, Morris DL, Liauw W, Bester L. Survival outcomes of a salvage patient population after radioembolization of hepatic metastases with yttrium-90 microspheres. J Vasc Interv Radiol 2010;21(10):1521-1526 